\title{
Airborne Trajectory Management for Urban Air Mobility
}

\author{
Capt. William B. Cotton ${ }^{1}$ \\ Cotton Aviation Enterprises, Inc., Lakeway, TX 78734, USA \\ David J. Wing ${ }^{2}$ \\ NASA Langley Research Center, Hampton, VA 23681, USA
}

\begin{abstract}
Urban Air Mobility (UAM) has captured the imagination of the public and the aviation industry for someday soon moving people and goods through and around metropolitan areas using Unmanned Aircraft Systems (UAS) that are electrically powered, environmentally friendly, and autonomously operated. Significant investment and rapid development of vehicles for this activity is taking place, with package delivery services already beginning in some areas. However, the ability to manage thousands of these vehicles safely in a congested urban area presents a challenge unprecedented in air traffic management. Initial studies of this problem led by NASA under the UAS Traffic Management (UTM) initiative have primarily focused on geo-fencing and centralized reservation of airspace for individual flights. This paper proposes an extension of UTM using a de-centralized approach employing airborne surveillance, self-separation, and a minimized "design separation" approach to permit the optimization and safety of each flight in very high traffic densities and close proximities. The concept employs Airborne Trajectory Management (ABTM) principles and a novel new concept for variable separation criteria to manage the angular velocity of a passing vehicle, thus eliminating the "startle factor" and perceived hazard of very close operations. ABTM also accomplishes most of the services required for safe planning and execution of normal flights and recovery from abnormal or emergency operations while accommodating conventional piloted flights using the current air traffic control paradigm. The environment for UAM operations is described along with the proposed means for autonomous, tactical separation of the vehicles. Sample geometries of traffic conflicts and resolutions are shown and the airspace definitions, rules for flight within them, and additions and exemptions to the rules for these flights are listed and explained.
\end{abstract}

\section{Introduction}

here has not been such a jolt to the public's imagination in anticipation of an aviation revolution since the post World War II promise of an aircraft in every garage. While that expectation did not lead to the envisioned end state, the drone revolution has in fact become reality at both ends of the size spectrum, and the level of interest and investment in solving the issues to get to the middle is unprecedented. There are already over a million small drones registered with the FAA (Federal Aviation Administration), mostly hobby quad copters, and the capability of the large military remotely piloted aircraft has been more than adequately proven in several theaters of conflict around the globe.

Out of these successes, many entrepreneurs worldwide are vying for a piece of a potentially much larger market, the use of Unmanned Aircraft Systems (UAS) for widespread delivery of packages, cargo, and goods of every imaginable kind and even people, riding in these vehicles without pilots, to their destinations. The potential manufacturers of these personal air vehicles include startups but also the likes of Boeing and Airbus, Bell and Sikorsky, JetBlue, Amazon, Google, Aurora Flight Sciences and Toyota. NASA has been very involved as well,

${ }^{1}$ Aviation Subject Matter Expert, wmcotton@gmail.com, AIAA Member

${ }^{2}$ ATM Research Engineer, MS 152, david.wing@nasa.gov AIAA Associate Fellow 
leading the industry efforts to define an appropriate means for managing the flood of new airborne traffic under the UAS Traffic Management (UTM) initiative. The FAA has been working to keep pace with these developments but, in the interest of safety, has so far only been able to issue a set of rules known as FAR Part 107 that pertains to small (less than 55 pound) UAS, limiting when and where they can fly. In general, they are limited to the line of sight of the remote pilot in daylight conditions below 400 feet above the ground and not over assembled people. Specific Certificates of Authorization (COA) and FAR Part 333 exemptions are issued to operators for more extensive uses of drones on an individual basis, but these will not enable the more revolutionary operations sought by the visionaries. At the end of the UTM Plan, Technical Capability Level (TCL) 4 is planned to address large numbers of vehicles in the airspace but that development is said to be years in the future, well beyond the time frame envisioned by the entrepreneurs.

Urban Air Mobility (UAM) has been called the "Third Aviation Revolution" by NASA researcher Irene Gregory. She very comprehensively sets forth the concept and the challenges to be overcome in order to realize this vision. [1] [2] Within the total spectrum of UAS operations in the National Airspace System (NAS), Urban Air Mobility refers to flight operations to carry people and cargo within the geographical limits of an urban metropolis. This is part of a broader view of this vision to carry "Anyone, Anywhere, Anytime". Within a city, however, the challenges are particularly difficult. Maintaining safety under all failure conditions while flying over people and property is particularly daunting. Launch and recovery areas must be located on rooftops, in parking lots, or on specially constructed platforms over roadways or other urban structures. While the vehicles are often described as flying taxis, it would not be feasible to step on and off at any street location as you would a current taxi, so the infrastructure cost is significant. The environment is filled with obstructions higher than many flights will cruise, and obstacles like construction cranes pop up and move on a daily basis making the obstruction database difficult to keep current.

A most significant challenge is that part of the dream hopes to solve the surface traffic congestion problem, and that promise for UAM is touted in many articles on the subject. For example, the metroplex chosen by Uber to test their UAM vehicle is Dallas/Fort Worth (DFW). This sprawling metropolis of 7.1 million people contains 2.5 million households, and each household has an average of two cars, meaning there are about five million cars in the area. More than 80 percent of the workforce drives to work, overwhelmingly alone in their cars. [3] In order to have any chance of noticeably reducing this surface congestion, at least 10 percent of those travelers would have to be lifted above the fray to their destinations. That means there is a potential for up to 500,000 air taxis/flying cars in and under the DFW Class B airspace, coming and going from thousands of locations on the ground, logging a million flights per day. This does not even count a far greater number of package delivery drones (under 55 pound) in the same airspace. By comparison, the DFW airport handles approximately 2,000 piloted flights per day that will still be there as well.

Clearly, for the Third Aviation Revolution to get off the ground, it will take more than building and certifying a very low cost, autonomous vehicle that is reliable enough never to fall on the people below. Because of the numbers of operations, the safety and reliability of the vehicles themselves will have to increase dramatically over current helicopters. Sikorsky Innovations Vice President Chris Van Buiten believes the autonomous flight system will have to "move from one failure per million hours to one in ten million hours." [4] Similarly, ensuring safety from mid-air collision with this density of air traffic while maintaining the ability to efficiently transport the people and goods to their destinations requires an entirely new concept for air traffic control for these vehicles. This paper sets forth a concept to accomplish that objective, based upon the principles contained in the Airborne Trajectory Management (ABTM) concept [5] [6] designed to accommodate large increases in conventional air traffic while improving both the capacity of the system and its operational safety. This concept provided for flight safety by de-centralizing the function (i.e., service) of managing trajectories of airborne vehicles, locating it instead in the vehicles themselves rather than in one or more ground-based service providers. This level of autonomy is anticipated to make UAM economically viable and invulnerable to catastrophic losses of service, to which centralized control systems are prone.

The current paper focuses specifically on the application of ABTM to the problem of UAM traffic management. Section II will describe the scope of the proposals for UAM operations and the physical boundaries within which these operations could take place. Section III will describe the basic principles of ABTM operations from the initial considerations for efficient, independent flight planning to the means for ensuring safety for all operators in the assumed very high density traffic environment. It includes detailed descriptions of the separation paradigms proposed and examples of representative encounter geometries and their proposed resolutions. Section IV contains a short summary of the proposals and recommendations for their development in order to handle the challenges attendant to dense, urban autonomous vehicle operations, and recommended research to validate these claims. 


\section{Scope of the Proposal}

This paper will use the airspace and surface features of the DFW metroplex, shown in Figure 1, as the area of study. The UAM area chosen is the region within the 30 mile Mode C "veil" centered at the south end of the DFW airport. The existing airports, flight patterns and traffic densities are assumed to continue with normal growth.

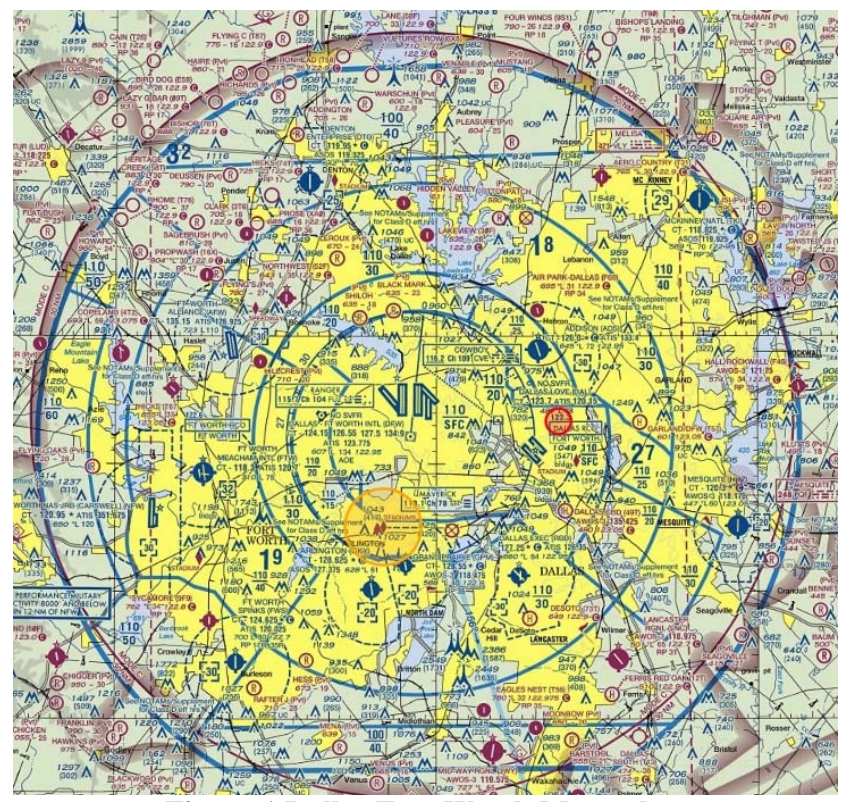

Figure 1 Dallas Fort Worth Metroplex.

Many of the electric or hybrid vertical takeoff and landing (eVTOL) vehicles under development have novel configurations, such as tilting wings or rotors, carrying moderate loads at faster speed over longer distances, perhaps 100 miles apart at altitudes up to 10,000 feet, but those are not the primary subject of this paper. Most vehicles proposed for UAM are small package carrying drones with limited range and endurance, and one or two person air taxi vehicles, proposed as urban shuttles connecting the downtown areas of cities with the close suburbs. None proposed so far are pressurized. The larger vehicles will be operating extensively in Class B, C, D and E airspace and, after an initial period in which they are piloted to establish vehicle safety, they are expected to interact autonomously with Air Traffic Control (ATC) and other manned flights in the airspace. The principles of ABTM, in particular the Autonomous Flight Rules (AFR) [7], would accommodate these operations in mixed airspace, both in their piloted and unpiloted operational phases. As these larger vehicles are very expensive, their numbers will be correspondingly small and thus manageable using AFR in conventionally controlled airspace where higher separation values are applied. This paper, however, is focused on the intra-urban area in which very large numbers of package and small personcarrying vehicles are proposed to significantly augment the surface traffic modes of transportation. Because of the expected numbers and density of this traffic, no traditional ATC is exercised in this environment.

The UAM area is up to 400 feet above the ground or above structures on the ground. As shown in Figure 2, wo classes of UAS are assumed in this description: a one- or two-person carrying vehicle (similar to the EHang 184 which has enjoyed recent successful flight tests [8]) and a 55 pound gross weight package carrying drone. Both are assumed to have multi-fan configurations with operating speeds from 0 to 60 knots for the cargo vehicle and up to 120 knots for the air taxi. As an aircraft category, they are neither helicopters nor airplanes. They are autonomously controlled for attitude stabilization and navigation and possess acceleration capability in all axes sufficient to counter anticipated turbulence and eddy currents that exist around tall buildings on windy days, while maintaining their planned flight trajectories. The UAS may operate from the ramp areas of the existing 28 public airports in the region and from roughly 50 downtown locations each, in both Dallas and Fort Worth, as well as at least 5000 designated spots in parking lots, parks, driveways and rooftops scattered throughout the 2,827 square nautical mile region under the Mode $\mathrm{C}$ veil.

Most UAM launch and recovery sites, called vertiports, would accommodate one vehicle at a time, but the downtown locations may have 20 or more spots for loading and unloading at a single location, sufficiently spaced laterally not to cause hazardous interactions between a landing/launch and an adjacent load and unload operation. The arrival, unloading,

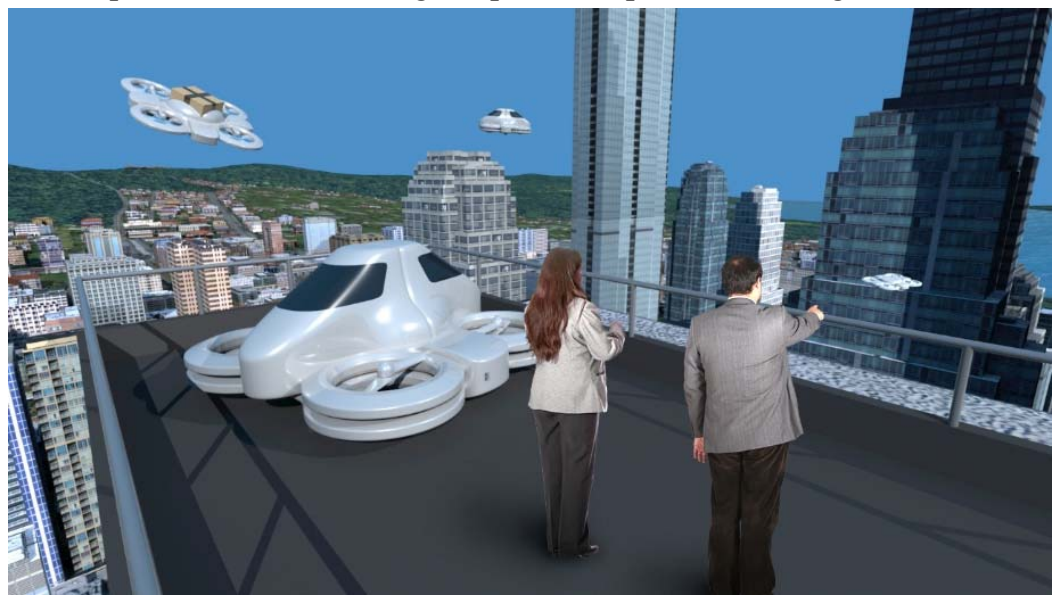

Figure 2 UAM will enable new vehicles to transport passengers and packages in dense urban environments. Graphic by J. Ponthieux. 
loading and departure of individual vehicles might be accomplished in as little as 30 seconds because the payloads are so small. Thus a 20 -spot vertiport on a downtown rooftop could theoretically handle up to 2,400 operations per hour. Passenger accommodations getting to and from the rooftop sites presents an obvious challenge but is beyond the scope of this paper, which focuses on airborne trajectory management.

All UAS are assumed to be equipped with Automatic Dependent Surveillance - Broadcast OUT (ADS-B OUT) capability, as will be required anyway within the 30 mile veil area after January 1, 2020, and with ADS-B IN that is used in the vehicles' Trajectory Management System (TMS). The TMS is onboard avionics that encompass the services/functions of flight planning, navigation, separation, and flight control. The navigation source for the TMS is augmented Global Navigation Satellite System (GNSS) and, for the land and launch operations, electro-optical visual surveillance sensing of the spot and its environs, including people and other objects that may inadvertently be in the way. Communications are digital, including initiation and termination of a flight by the operator, and autonomous from the vehicle to the operator as required to report abnormal and emergency situations. Communication among UAS is normally performed passively via ADS-B IN and OUT. The assumed UAS traffic is split 80/20 between small package delivery and human carrying flights. The maximum airborne UAS traffic count in the DFW UAM area is assumed to be 100,000 . As UTM research to date has not ventured into the management of UAS traffic at this density, this paper explores the feasibility of using the ABTM concept as a means for achieving safety and efficiency in this TCL 4 environment, thus extending the UTM research and development activity to date.

\section{ABTM Traffic Management Principles for Achieving UAM}

This section discusses the principles of traffic management employed in the ABTM concept that are proposed to solve the problem of maintaining safety in the presence of the assumed UAM traffic density. The principles are:

- Plan each flight independently on the most efficient route, avoiding obstacles and protected airspace

- Dynamically geo-fence airport runways and other protected operations

- Ensure separation and collision avoidance among proximate vehicles, using:

o Small separation values and self-separation

o Strategic, flexible navigation plans and tactical separation

o Horizontal, vertical, and speed (including stop) conflict resolution modes

o Interval management close to common destinations

o Counterclockwise de-compression of many converging aircraft

o Implicit coordination for separation; explicit coordination for collision avoidance

- Apply comprehensive traffic operating rules

- Ensure spot availability before landing or initiating launch operations

Each of these principles is discussed separately below.

\section{A. Flight Planning}

The operator's only role in flight planning involves the routing and dispatching of the vehicle. Cargo operators will transmit the destination from their dispatch center to the vehicle when loading is complete. Passenger carrying vehicle flights will be initiated by the operator, but the destination will come from the customer at the time of flight request. Destinations will be vertiports and eventually street addresses. Any address in the local UAM database will be an accepted destination if it is within range of the vehicle with reserves (to get to a re-charge point) and has been approved with a "launch spot" designation. Launch spots will be marked with a distinctive painted symbol, recognizable by the UAS vision system, and may also have electronic identification for authentication, similar to a toll pass sticker on a windshield.

The automated flight planner in the vehicle then performs a best wind route and altitude search from present position to the selected destination. Altitudes used in flight are sensed by augmented Global Positioning System (GPS) rather than barometric altimeters, and referenced to mean sea level. In that narrow vertical band from the surface to 400 feet above the surface, the altitude used in flight will be a smoothed height above ground level (AGL), calculated from the navigation data base, and will rarely be level because the height of the ground itself varies considerably over short distances in most locations.

Most of the range of altitudes from 100 to 400 feet above the local surface is within the surface boundary layer of wind. This means that wind direction and velocity can vary considerably over this short vertical distance. When the best wind trajectory is found, it is compared to the real-time protected airspace database and modified to prevent incursion into any static protected areas. Protected airspace includes both static (all the time) and dynamic areas containing higher priority operations, such as active final approach and initial departure paths from airports with manned flights in the region up to 400 feet AGL, as well as other designated sensitive locations, protected for security 
reasons, such as active police or fire fighting operations. The protected areas are geo-fenced for avoidance by the UAM flights.

The planned trajectory is also checked against terrain heights and obstacles in the terrain and obstacle database. A new, detailed database for the DFW 30 mile veil will be created for the purpose of UAM and will contain building heights, towers, wires, lone trees, and construction cranes, composed of the height of the highest obstacle in each cell of a 10 meter grid. It will be much more comprehensive than the detailed surveys conducted for airport instrument approach procedures in order to permit flights to and from any address in the region. Maintaining a safe height above the ground and obstacles when the choice of altitudes is only 300 feet deep is not trivial, especially if not equipped with radar altimeters, as these vehicles are not expected to be. The process is very database dependent and should be visualized as draping a very large blanket over the whole region in which the trees, towers and buildings are visible as points holding up the blanket at their locations and the blanket slopes away from the peaks and smoothes the surface in between. After a 100 foot vertical launch, the vertical component of the trajectory stays between 100 and 400 feet above the blanket at a height where the wind is most advantageous. Uncharted obstacles, such as a crane that was erected without following the approved process, or large birds that do not care, must be avoided tactically using the short range radar and visual systems on the drones. This is an important consideration, as the buzzards that number in the thousands in the DFW UAM area could damage both the lift fans in a two-fan corner vertical stack if they were hit, resulting in loss of control of the vehicle.

Weather planning for UAM is quite different from that performed for conventional manned aircraft. The whole region of interest is only 60 miles across and only contains altitudes from the surface to 400 feet. As these vehicles are autonomous, ceiling and visibility are not an issue. A Category III visibility of 300 feet is sufficient for the electrooptical and radar sensors to ensure the landing spot is clear of hazards and to "see and avoid" any unmapped obstacles in the path. Wind, hail, and freezing precipitation are hazards to be concerned with, however, and automated microprognoses for the area in question will become an important science/art. For the 20-30 minutes into the future that for most of these flights is near the battery limit, forecasts for these small areas can be quite precise by location and accurate in expressed meteorological parameters. It is expected that automated weather sensors, such as those in place at most regular airports today, will be optimized for the UAM needs and placed at very many additional locations around each metro area having UAM operations. As forecasting algorithms will be created to project the values for wind and precipitation in 10-minute increments out to 30 minutes ahead using data from the automated reports at each location, the whole ABTM planning process, including navigation, obstacle, protected area, and weather avoidance, can be automated within the vehicle. When a path thus de-conflicted from reserved airspace, obstacles, and the weather checks OK for the flight, a "ready" light illuminates by the operator and the flight may be launched with a single button push.

Just like automobile surface traffic, the trajectory plan only exists for the operator and the vehicle. There is no centralized control or oversight except for the promulgated rules of operation listed in an extension (and exceptions) to FAR Part 91. Not all flights are point to point, of course, as surveillance missions in all forms and even sightseeing will be popular uses of UAS, departing from and returning to the same point. As these autonomous vehicles are programmed to operate in compliance with the rules, the safety issues on the roads caused by human drivers not following the rules will not exist. If unforecasted weather conditions are encountered, beyond the prescribed operating limitations for the vehicle, it will return to the origination point if the path there is safe, or terminate at the nearest safe launch spot and advise the operator.

\section{B. Dynamic Geo-fencing}

All the runways at the 69 airports in the area have manned flights taking off or landing in the airspace below 400 feet. Some, such as DFW, are in nearly continuous use and many others have far less use. During takeoff and climb up to 400 feet AGL and for the last 400 feet of descent to landing, these manned flights will "own" that airspace defined by geo-fenced corridors into which the drones cannot operate, as shown in Figure 3. Most of this fencing is dynamic in nature, making the airspace available when not otherwise in use. Since these areas may be occupied by a drone at the time of their activation, they are narrow enough that they can be vacated within 30 seconds of activation. Helicopters also use the low

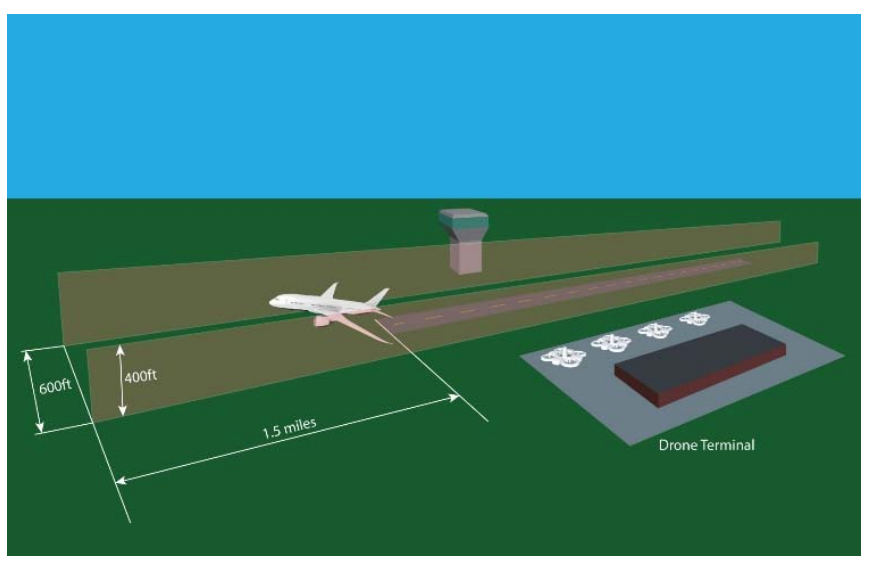

Figure 3 Dynamic geo-fenced approach and departure areas segregate UAM operations from ATC-controlled flights at airports. 
altitude airspace but their numbers are small enough that they may be detected through the normal surveillance means and given right of way by all UAS, according to the AFR rules.

\section{Separation and Collision Safety \\ 1. General}

The vehicle automation provides self-separation and collision safety from other flights using onboard surveillance and algorithms for conflict detection and resolution (CD\&R). Even though the Class B airspace goes to the surface in the inner circle, there is no centralized air traffic control exercised in the region below 400 feet AGL and outside the protected areas. The cargo and passenger vehicles providing UAM in the manner described in the scope of this paper will operate almost exclusively within this airspace. No piloted aircraft except helicopters will operate within this airspace because they would be below the regulatory minimum altitude for flight over populated areas. In such airspace, piloted aircraft are only allowed to operate that low when landing or taking off. That is why protected areas would be defined off the ends of runways for about 1-1/2 miles to accommodate these operations. Some of the eVTOL aircraft under development are intended for operation to and from regular airports within the metropolitan area and for flights well beyond the 30 mile veil examined in this paper. When such a UAS arrives at or departs a regular airport within the fenced area, an ATC clearance must be obtained just as for the manned flights operating in that airspace.

The in-scope UAM flights described here are also "independent" flights. An independent flight, in this sense, means not only that responsibility for separation and all other safety rests within the vehicle, but also that the vehicle is not in a cooperative mission with another vehicle. Cooperative UAS missions are many and varied but relatively rare in the UAM environment. Such missions have been shown to be capable, for example by Intel at the 2018 Winter Olympics where over 1200 vehicles performed together, of dynamically establishing and maintaining very small but precise relative positions with respect to each other, and absolute positions with respect to their surroundings. Intel did this not with vehicle to vehicle communication, but with all vehicles being commanded from a single computer to take up their appropriate place in an earth-fixed reference frame for that "pixel" in the drone light show display.

An independent UAM flight uses its strategic navigation mode throughout, probing and updating the most efficient wind trajectory to the destination, perturbing that path only as necessary for conflict resolution, abnormal, or emergency procedures. The surveillance used in the vehicles is primarily ADS-B IN, with the capability of increasing its "heartbeat" rate to $120 \mathrm{~Hz}$ when in conflict with another vehicle, or when performing interval management relative to another vehicle for arrival at the destination point. The surveillance will also include electro-optical "vision" and short range radar sensing (like on self-driving cars) on some vehicles for obstacle avoidance and close-in navigation at the launch point. This combined surveillance system can provide sub-meter accuracy when at close range.

\section{Separation Concept}

The ABTM concept of separation for UAM is quite different from that used in conventional ATC. In ABTM for UAM, the entire control loop (i.e., sensing, algorithmic processing, and resolution maneuvering) is automatic. There is no human reaction or cognitive time in that loop, and the process is entirely deterministic. As the process is completely performed by software in the vehicle with the output directly controlling the trajectory flown, the nominal values of distance and time between vehicles common to a radar-based, human-centered ATC system are no longer relevant. What is commonly referred to as "minimum legal separation" is replaced in this concept by "design separation," the value of which is variable rather than fixed. The longest time from detecting a conflict to the closest point of approach that must be accommodated to ensure separation in this concept is defined as 10 seconds, so the process should be thought of as tactical separation. All degrees of freedom are used in the separation and collision avoidance maneuvers: lateral, vertical, and speed, including stop, with the design acceleration values low enough not to alarm the passengers.

The assumed density of UAM vehicles in the area demands that they can be safely operated very close to each other. The high accuracy and low latency of the integrated surveillance systems proposed in this concept would support a minimum value of 50 feet design separation, both lateral and vertical, between the extremities of the vehicles at low relative velocities or when performing interval management. Since barometric altimetry cannot support a 50-foot vertical separation, Space Based Augmented GPS (SBAS) is used for height as well as lateral position determination. The minimum separation distance is supported by a Required Surveillance Performance (RSP) standard, similar to Required Navigation Performance (RNP) used on certain Area Navigation (RNAV) routes. While the 50-foot minimum design separation is recognized as a stretch goal, experience gained at a larger value could validate whether this goal can be achieved. It should be noted that autonomous automobiles can beat this goal today, at comparable speeds. 


\section{Variable Separation}

In attempting to wrap one's mind around the feasibility of this concept, consider that as long as the flight paths can be precisely controlled, this very close separation is still larger than what is common when driving, even driving at high speed in opposite directions on two lane roads, as autonomous cars do today. However, the 50 -foot minimum between air taxis carrying passengers could be very unnerving except in very low closure rate scenarios, so it is proposed in this concept that a maximum angular velocity of the bearing to the target be established such that a passenger in an air taxi will not see another UAS "whipping by" at an unnerving rate. The angular velocity requirement is what makes the design separation a variable rather than fixed distance. For automobile traffic, the white line down the center of the road provides the comfort for high speed, opposite direction encounters. In flight, without such a reference divider, larger distances based on the maximum acceptable angular rate of bearing change to the target could provide the comfort. A value of one radian per second could be a starting value for simulations designed to determine an acceptable value for this parameter. The one radian per second value provides a simple relationship between the closing speed of the aircraft and the lateral separation provided at the closest point of approach (CPA). As shown in Figure 4, the minimum

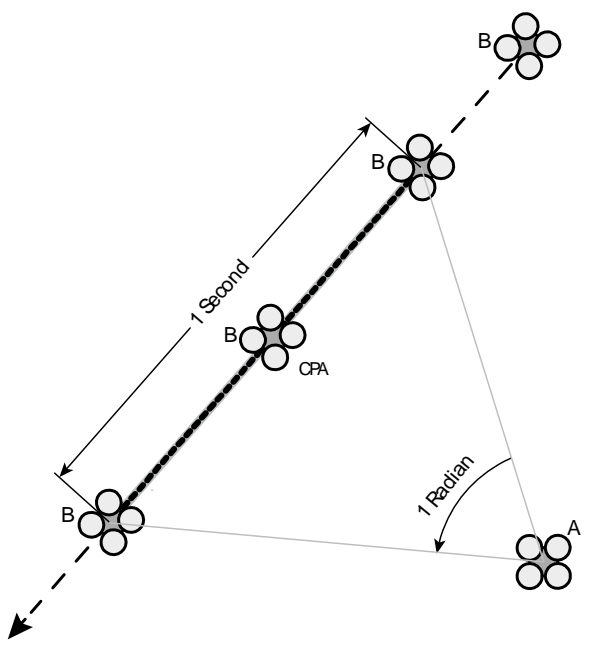

Figure 4 Maximum angular velocity at the CPA is designed to not exceed one Radian per second. separation distance will be the same numerical value as the initial closing speed. For example, head-on traffic closing at 120 knots (203 feet per second) would be separated by 203 feet laterally at the abeam point to pass at 1 radian per second. This additional "adaptive" design separation standard would not be needed or used by all cargo UAS, as there is no one on board to be alarmed by what looks like a "near collision" but is actually controlled separation.

Thinking of a hundred aircraft within a square mile of airspace sounds unmanageably crowded to those accustomed to conventional air traffic management, but it is wide-open space compared to the density of cars in rush hour traffic. The most important facet of ABTM for UAM is that, using its automated and adaptive separation paradigm, there is no need to organize traffic flows along virtual highways in the sky because there will still be relatively few aircraft compared to the size of the airspace when this very close design separation is used. In [4], the Uber proposal for UAS corridors in the DFW metroplex undermines the point of UAM flight. That is, they should not take fixed routes that follow basic cardinal headings between 10 stations, but instead fly point to point among thousands of points within the area representing the actual locations where people and goods wish to travel, the UAM goal of end-to-end transportation. It is likely that launch and landing points for the small package vehicles will be easier to get approved at thousands of locations than for the larger, noisier passenger carrying drones, but there will still be thousands of such spots. Therefore, the strategic flight trajectory for each vehicle should be optimized for the existing wind from present position to the destination. The trajectory can be flown by each vehicle with only minor perturbations occasionally needed to resolve conflicts with other aircraft. It works because the design separation (50 feet or $1 \mathrm{rad} / \mathrm{sec})$ is so small. The high altitude skies over the northeast may seem crowded with airliners today but that is because no aircraft can get closer than five nautical miles to any other, and actually being as little as five miles apart is rare. That practice reserves 113 million square feet of airspace for one aircraft versus 7,854 square feet for a UAS using this paradigm; this represents over a 14,000 times reduction in the airspace required for separation.

4. Separation Logic

The ABTM separation logic providing both a minimum distance and a maximum angular velocity between human carrying aircraft uses integrated airborne surveillance as its input and analyzes encounter geometries in both an earthfixed, and for heading determination, an air mass reference frame, as follows. For all traffic within 1 mile from the "ownship," establish a data record with range, range rate, altitude, altitude rate, bearing, and heading angle. Calculate Tau $(\tau)$ (equal to range divided by range rate representing the time to the closest point of approach, or CPA) and, for all targets with $\tau=12$ seconds or less, increase the ADS-B surveillance rate to $120 \mathrm{~Hz}$. Then calculate the projected vertical separation $\mathrm{v}$ at the closest point of approach (CPA) and the magnitude and bearing of the projected horizontal miss distance, 1 , at the CPA. If $\mathrm{v}$ is within 100 feet of the ownship trajectory altitude in $\tau$ seconds, calculate the angular velocity $\omega$ of the relative bearing at the CPA. If it is greater than $1 \mathrm{rad} / \mathrm{sec}$ or the projected miss distance is less than 50 feet, a conflict exists with that target. Compare the target heading to that of ownship to determine which aircraft 
has right of way using the values in Error! Reference source not found.. If the target has right of way, calculate the required miss distance based on the closure speed to make $\omega=1$. Then calculate how much additional miss distance is needed to make $\omega$ equal 1 at the CPA, or 50 feet, whichever is greater. This miss distance will be the design separation for this particular encounter. Initiate a lateral turn when a $1 / 8 \mathrm{G}$ acceleration will create the needed separation at the time of CPA. If ownship has right of way, monitor the projected miss distance to ensure that the target is providing design separation.

It is proposed that a new airspeed limit be imposed at and below 400 feet AGL within the veil, equal to 120 knots. This value is chosen as it is near the maximum cruising speed of a typical passenger carrying autonomous UAS and provides a limit for required surveillance. The encounter requiring the largest lateral separation when the target is abeam in order to equal the one radian per second maximum angular velocity is a head on conflict between two vehicles, each with an airspeed of $120 \mathrm{~K}$, thus a closure speed of 240 knots ( $405 \mathrm{fps}$ ). The most critical geometry is an initial predicted collision, requiring that the greatest displacement from current track be accomplished in the remaining time to the CPA, as shown in Figure 6. The logic requires that a standardized resolution maneuver based on the encounter geometry be initiated when the predicted time to closest approach equals the time needed to establish

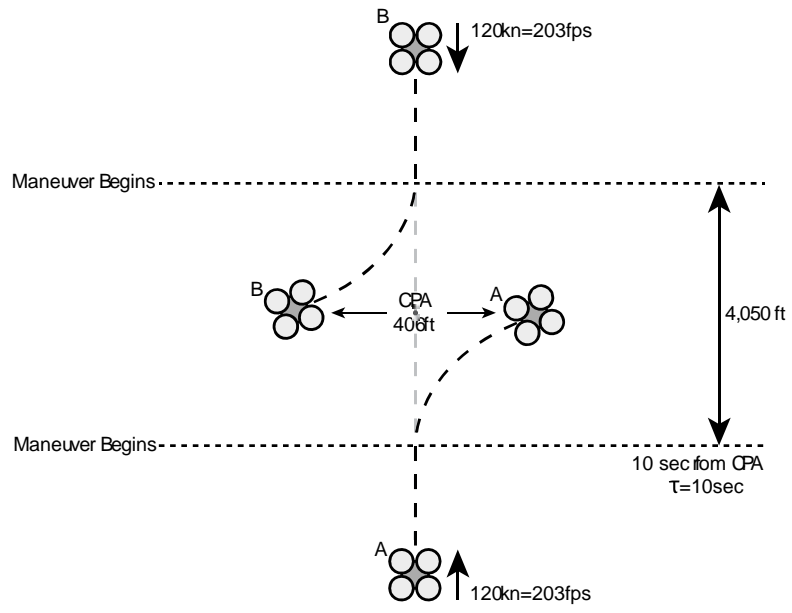

Figure 6 A head-on encounter represents the worst-case horizontal condition that drives the detection time horizon.

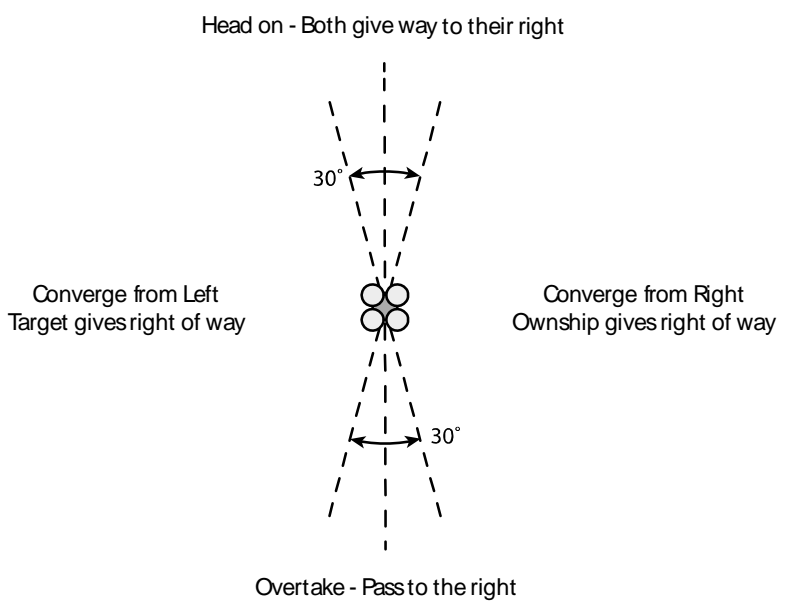

Figure 5 Right of way in converging conflicts is based on the bearing to target. the design separation. All autonomous vehicles operating in the UAM airspace would be required to use the same separation logic (like TCAS) so both vehicles in this headon encounter, each required to alter course to the right, would begin to react within one second of each other, each using a standard $1 / 8 \mathrm{G}\left(4 \mathrm{fps}^{2}\right)$ acceleration to the right. Each vehicle must move half the distance ( 203 feet) by the time of passage. This time is given by the square root of 2 times the distance divided by the acceleration $\sqrt{ }(2 \times 203 \div$ 4) $=10$ seconds. In this worst-case scenario of a 240 knot closure, the maneuver would begin when the vehicles are 4050 feet or $2 / 3$ mile apart. It may be seen that using this ABTM tactical separation logic, the airborne surveillance range need only be one mile to track and predict the furthest conflicting aircraft. Conflicts between vehicles not carrying humans need only meet the 50 -foot design separation criterion, generally requiring a lot fewer resolutions (i.e., big sky effect) and even closer-in maneuvering.

To review, ABTM tactical separation is designed to permit the highest density of traffic and a minimum of resolution maneuvers. It is based on the following process for conflict resolution: a resolution maneuver to ensure design separation begins when the value of Tau $(\tau)$, equal to the absolute range to the target divided by range rate, is equal to the time required to establish design separation at the closest point of approach using standard resolution maneuvers (subject to validation) of $1 / 8 \mathrm{G}$ lateral acceleration, $1 / 8$ $\mathrm{G}$ longitudinal deceleration or $1 / 4 \mathrm{G}$ vertical acceleration. If, because of the encounter geometry or low vehicle speed, the closure speed between the aircraft in conflict is 50 feet per second or less, a minimum design separation of 50 feet between the vehicle extremities is used.

The surveillance data includes the augmented GPS position including the GPS altitude and the velocity vector for each target, as well as short-range radar and optical sensors. The velocity vector is measured in an earth fixed reference frame and is used in the calculations of $\tau$ and the projected miss distance at the CPA. The reason for exchanging heading between targets in conflict is that it is used in the right of way rules designed for piloted aircraft in which pilots looking out the windshield observe the convergence angle with respect to the nose (i.e., heading) in determining right of way. This same reference exists for passengers in UAM air taxis who would perceive the encounter in the 
same way. Aircraft track would be used in the separation logic for greater stability but right of way must be calculated the same in all vehicles to ensure implicitly coordinated resolutions take place.

In addition to $\tau$, the predicted horizontal and vertical miss distances are calculated and used in the separation logic to decide which way to turn if a lateral resolution is chosen. Track stability is watched to determine if the target is turning. If it is, a vertical resolution is chosen because without knowing when the turn will stop, the horizontal miss calculation is unpredictable. The vertical resolution takes account of any existing climb or descent by the target. Vertical rates and displacements will be quite low for these aircraft because the navigable airspace is very limited in that dimension. Longitudinal separation is chosen by the logic when the aircraft are converging slowly on similar headings to achieve the required miss distance at CPA without changing the course to destination. Collision avoidance takes place if the design separation is not being achieved as designed, and adds any and all maneuver dimensions by both aircraft, coordinated to be compatible, to increase the miss distance at CPA.

\section{Additional separation examples}

Several additional examples will help to illustrate this concept. In Figure 7, two 55-pound box carriers are converging at the same altitude at a 90 degree angle, each with an airspeed of 40 knots. Assume that without maneuver, the targets will collide at the trajectory crossing point. Because there are no people aboard, the design separation of 50 feet between extremities may be used, and assuming both vehicles are 4 feet across, 54 feet between centers must be achieved. In this geometry, vehicle A must give right of way and achieve a displacement of 54 feet at the closest point of approach. The box carriers have a capability of $1 / 4 \mathrm{G}$ lateral acceleration $\left(8 \mathrm{fps}^{2}\right)$ because passenger comfort need not be considered. Using the same formula as above, $\tau=\sqrt{ } 2 \times 54 \div 8=3.7$ seconds is required to establish the design separation of 54 feet at the time the drones pass and begin to separate. The closure speed is 40 knots divided by the Cosine of 45 degrees, or 57 knots $(96$

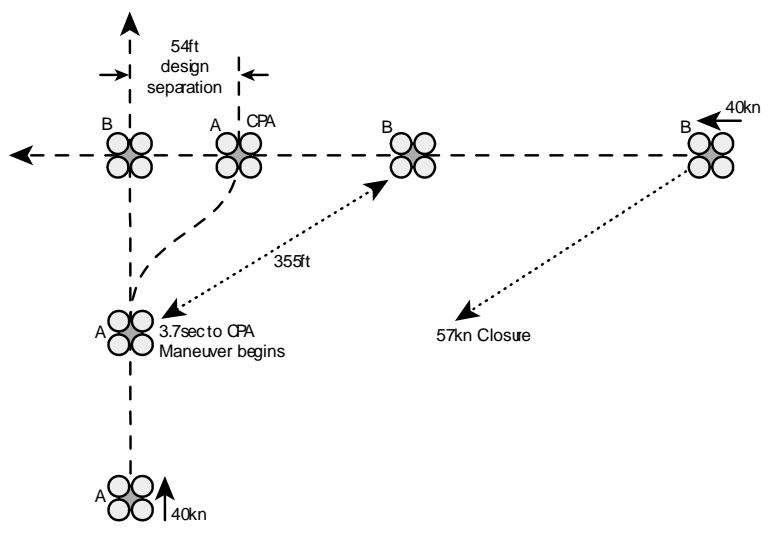

Figure 7 In a conflict encounter with convergence from the right, vehicle $A$ gives right of way. fps). When the resolution maneuver begins at 3.7 seconds to closest approach, the aircraft are 355 feet apart. At the $\mathrm{CPA}$, vehicle A passes 54 feet behind vehicle $\mathrm{B}$, then continues to its destination.

In Figure 8, two air taxis are converging on nearly parallel tracks, each flying at 60 knots. The 15 -degree convergence angle produces a closure speed of 16 knots $(27 \mathrm{fps})$. Because of the low closure speed, angular velocity is not an issue in this encounter, but as these vehicles are 25 feet across, they must pass with a design separation of 50 $+25=75$ feet. In this geometry, vehicle B has right of way, and vehicle A must establish the 75 feet at closest passing.

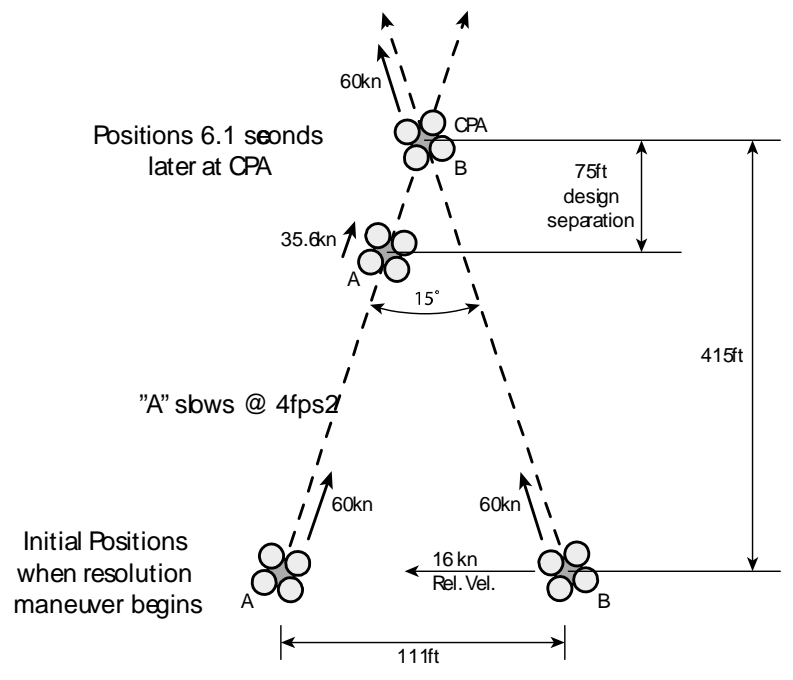

Figure 8 In a conflict encounter with nearly parallel converging geometry, vehicle A reduces speed to allow vehicle $B$ to pass.
If vehicle A turns to the right, design separation would be lost. If vehicle A turns to the left, the flights will be parallel and vehicle $\mathrm{A}$ will not get to its destination. Therefore, vehicle A must slow its speed. A $1 / 8 \mathrm{G}$ deceleration will create a 75-foot miss distance in 6.1 seconds. At the beginning of this maneuver, the two aircraft are 415 feet from where they would converge and 111 feet apart laterally. These drones are capable of speeds down to zero and may use this flexibility, if necessary, in the resolution logic.

Minimum vertical separation is proposed to be 50 feet between aircraft in a tactical conflict resolution, but cruising flight may occur at any altitude from 100 feet AGL to 400 feet AGL, and altitude is read as augmented GPS height above sea level to provide a common, fixed reference frame for this measurement. The heavier UAS will not be able to pass each other at 50 feet vertically because their downwash may cause loss of control of the vehicle below. The use of level cruising altitudes is impractical because there is not enough room between the 
surface and 400 AGL for it to work. The land is fairly flat in the DFW area where the field elevation is 607 feet. But airports within the 30 mile veil have elevations from 447 feet in the east to 855 feet in the western part. Other terminal areas are not nearly that flat so the drones will have to climb and descend almost continuously just to stay between the 100 and 400 foot levels above the surface. Many buildings and towers in the downtown area have tops more than 400 feet above the terrain, of course, so the vehicles will generally pass these to the side unless they are landing on the top. One building in downtown Dallas is 939 feet high and a TV antenna south of DFW, well within the veil, reaches 1640 feet above the ground with guy wires extending out all around it. Because of these factors, it is proposed that most resolutions be performed with a lateral path change or a speed change. The vertical dimension should be used when the target is in lateral non-linear flight or for collision avoidance when design separation has been lost, if, for example, a failure has prevented the normal separation system from producing the designed result.

\section{Dense Traffic Convergence}

The ABTM paradigm for traffic management permits large volumes of aircraft to operate in a relatively confined airspace and without structured routes either laterally or vertically. There may be times when specific locations under the veil have exceptionally large volumes of aircraft converging on them simultaneously. Some of these may be approaching destination addresses close together and others may be overflights funneled between geo-fenced areas. Without a mechanism like flow control, used to limit the volume of piloted flights at a busy terminal area, ABTM for UAM uses a mechanism to marshal the "flows" from all directions into a counterclockwise spiral around the point of greatest dynamic density by biasing the individual conflict resolutions to the right when more than one conflict exists in a single vehicle at the same time. Previous research has shown that this type of behavior results naturally from use of the right of way rules by all aircraft. In the extreme, this bias could be coupled with interval management at the minimum design separation among all the vehicles within the same portion of what would appear as a spiral flow. Those aircraft that are overflying the convergence would also climb to the maximum altitude of 400 feet AGL, while those arriving within the area would descend and spiral down to their individual destinations. Coming to a stop and hovering is always an available option for the separation logic.

\section{Operating Rules Considerations}

Self-separation is an automatic function in the aircraft built around a complete set of rules covering all possible encounter geometries, flight phases, and missions. By using these rules, it can work without explicit coordination communications among the vehicles themselves. This practice, called implicit coordination, was defined and coded into the self-separation research software developed at NASA Langley Research Center. [9] Having the location and velocity vector of other nearby aircraft is sufficient to detect conflicts and compute resolutions to conflicts in an unambiguous way using implicit coordination. The common view of the local traffic picture from surveillance in each aircraft permits ABTM tactical self-separation to function with integrity as presented in the last section using rulesbased, implicit coordination.

Still, a number of Federal Aviation Regulations must be changed or created, and certain UAS operations must be exempted from some FARs to enable the described UAM operations. For example, the vehicles themselves do not fit into any existing aircraft category, so a new one has to be created with appropriate aircraft certification standards. The vast majority of vehicle designs use lifting fans that are not helicopter rotors. The blades are fixed pitch so autorotation in the event of power loss is not possible. This takes them out of the rotorcraft category.

Safety in a power loss situation is important, and the solutions are not trivial. In a four-fan configuration, loss of any one fan makes the vehicle uncontrollable. For this reason, all the passenger carrying vehicles under development using the "four corner" configuration have two, stacked, independent fans at each corner, with either one capable of supporting its share of the vehicle weight. Many of the small box carrier drones may not have this safety feature and since they cannot glide like a winged aircraft, they must have a parachute system to prevent hazardous falls to the surface in populated areas. Unlike the popular CAPS (Cirrus Airframe Parachute System) on Cirrus aircraft, the drone parachutes must open very quickly with almost no altitude loss. They can be designed to do this because at their slower speeds, rapid opening will not cause a deceleration that rips the parachute from the vehicle. The descent should also be controlled using gliding parachute technology and electro-optical guidance to a "least hazard" landing spot. Additionally, an aural siren might warn people below to look up and get out of the way.

Rules for operating in the UAM airspace are simplified by the almost complete absence of piloted aircraft except for helicopters and airplanes in protected (i.e., geo-fenced) locations. Inside the DFW veil within 400 feet of the ground, there is Class B, D and G airspace, each with its own operating requirements. Class B operations, in addition to avionics equipment requirements, require that flights be on an ATC clearance. This is clearly impractical for the predicted volume of UAM vehicles, so geo-fencing to protect the airspace in the approach and departure paths of runways up to 400 feet AGL will have to be imposed for about 1-1/2 miles off the ends of the runways. Using the 
Terminal Instrument Procedures Standards (TERPS) obstacle protected lateral dimension of 300 feet to the side of the extended centerline, the protected, geo-fenced area would be as pictured in Figure 3 and in place (i.e., promulgated electronically to all UAM vehicles) when actually in use by a piloted aircraft. For departures, this would begin when a flight is cleared for takeoff (or announces departing at a non-towered airport) until ADS-B surveillance (required on all aircraft under the veil) detects it above 400 feet AGL and climbing. For arrivals, the airspace is protected from the time it is cleared to land until touchdown. At non-towered airports, this condition is learned from the Common Traffic Advisory Frequency (CTAF) transmission corresponding to an aircraft descending below pattern altitude on base or final.

There is still plenty of room for drone access into the sides of airports in the Class B area (including DFW and Dallas Love Field (DAL)) for passenger connections and cargo drone use to and from the airports, but they will have to be exempt from the rule requiring an ATC clearance. The 11 Class D airports with control towers, but underneath the Class B airspace, would be handled the same way. The approach and departure areas would be geo-fenced when necessary, and access to the airport by UAM vehicles would be from the sides. At the dozens of Class G airports inside the veil, there is no tower or CTAF to maintain the geo-fenced areas, but if the UAS avoid the runway extensions when surveillance shows them in use, they may also gain access to these airports from the sides of the runways. This rather simple accommodation is possible because of FAR 91.119, Minimum Safe Altitudes - General. "Except when necessary for takeoff or landing, no person may operate an aircraft below the following altitudes,... over any congested area of a city, town or settlement, or over any open air assembly of persons, an altitude of 1000 feet above the highest obstacle within a horizontal radius of 2000 feet of the aircraft". This keeps the manned aircraft above all the UAM aircraft (exempted from this rule) in all but the geo-fenced areas immediately off the ends of runways. Over other than congested areas, airplanes may be operated down to 500 feet AGL, still 100 feet above the UAM area. They may go lower than that over "sparsely populated areas" but these do not exist under the 30 mile veil.

However, the minimum safe altitude rule provides an exemption for piloted helicopters for their operations. They may fly below the prescribed 1000 foot and 500 foot minima "if the operation is conducted without hazard to persons or property on the surface." Further, "the helicopter [must] comply with any routes or altitudes specifically prescribed for helicopters by the FAA." Because the numbers of piloted helicopters in the UAM airspace will be very small by comparison to the drone population, they may be given right of way in all conflicts with drones but should be subject to the same small design separation values.

Whether the public will accept UAM vehicles operating much lower than current aircraft are allowed remains to be seen. The same goes for launch and landing in residential or populated downtown neighborhoods. These operations may be found acceptable if the vehicles are not very loud (a real challenge for fan vehicles weighing several thousand pounds) and they perform the first and last 100 feet of flight vertically. Public acceptance would also be facilitated by the use of ABTM and autonomous vehicles.

The standard resolution maneuvers employed in the ABTM tactical separation system begin with the existing right of way rules for manned flights found in FAR 91.113, but are modified for the unique characteristics of these UAS, such as their ability to stop, and specified numerically where generalizations are used. When converging, the aircraft on the right has the right of way. When approaching nearly head on, each vehicle alters to the right. An overtaken vehicle has the right of way and the overtaking vehicle alters course to the right while passing. Landing has right of way over other operations and the lower aircraft on approach has right of way. FAR 91.113 also prescribes that when giving right of way, you shall not pass over, under or ahead of the other vehicle unless well clear.

These right of way rules were designed for piloted aircraft. When codified into software, the words "or nearly so" and "well clear" must be numerically specified as the RTCA committees for UAS and other NASA studies have already found. [10] [11] The same is true for the words "nearly," "converging," and "overtaking." Specific values for the angle between the headings of two aircraft in conflict must be specified and codified into the separation software for implied coordination to work well, and the right of way of a hovering vehicle needs to be added to the rules. While the optimum values for these distinctions should be determined through analysis and simulation, representative values generally used by pilots of manned aircraft are as follows. Headings within 30 degrees of equal can be considered same direction (for overtaking encounters) and within 30 degrees of 180 can be considered opposite direction. All other relative headings are considered converging, as shown in Figure 5.

The part of the rule prohibiting passing over or under should not be applied to autonomous UAS as there is no issue with "losing sight" of the target, which was the reason for creating it. However, at the minimum vertical design separation, the heavier vehicle must pass beneath the lighter one to prevent wake upsets. Also, the part of the rule giving landings priority does not apply because with vertical operations from a pad, any vehicle on the pad must depart before it can be used for a landing, so right of way goes to the departure.

One other existing rule related to right of way that does not relate well to UAM is FAR 91.111, "Operating near other aircraft." Intended to address Visual Flight Rules (VFR) flight of piloted aircraft, the rule states that you may 
not operate so close to another aircraft as to cause a collision hazard. Since the UAS in the reference airspace are all exercising rules-based self-separation, the idea of "operating to cause a collision hazard" is absent by definition. The rule goes on to prohibit formation flight (except by arrangement), and no formations are allowed when carrying passengers for hire. The UAM air taxis are only carrying people for hire, and interval management formation flight in certain dense traffic situations may be the norm, thus an exception from FAR 91.111 is required for UAM operations.

\section{E. Spot Availability and Local Traffic Control}

If two or more vehicles are approaching the same landing address, their arrival order is determined by the earliest Estimated Time of Arrival (ETA) at the site. If there are multiple pads at a single site, access to the final longitudinal 300 feet of the approach will be along the radial assigned to each pad, and the last 100 feet of descent will be vertical so that a previous departure can use the same radial 50 feet above. A third party service providing automated pad assignment and arrival sequencing at multi-pad vertiports will surely emerge to meet the need as traffic builds to a level requiring it. The Micro Tower developed and used at Potomac Airfield southwest of Washington, D.C. is an example of this private development model. [12] Using voice recognition, artificial intelligence, and synthetic voice response, it provides basic weather and tower services to piloted aircraft using this airport. It would be much easier to provide sequencing, landing, and launching clearance to other computers located in autonomous UAS without having to interpret and respond to human language. A similar model exists in AirMap, a third party UAS Service Supplier (USS) for drone pilots. They have been granted authority by the FAA to provide nearly instantaneous airspace approval authority to operate drones near 500 airports nationwide using a service called Low Altitude Authorization and Notification Capability (LAANC). This service ends the previous laborious and time consuming application and approval process. Extending USS to local control at vertiports could easily follow.

\section{Summary}

While commercial investment in the development of vehicles that might be used in UAM operations is already approaching a billion dollars, and UTM research at NASA begins to address the integration of these operations into the NAS, relatively little attention has been paid to how thousands of these vehicles can safely operate in urban areas without collision or passenger alarm, yet still retain efficiency of flight in this confined environment. Airborne Trajectory Management is proposed as a potential solution to both objectives, combining minimal, rules-based airspace management with small-value design separation performed by on-board surveillance and CD\&R for selfseparation. The primary existing air traffic rules requiring alteration or exemption to permit UAM flights were identified along with the needed changes. It is concluded that very high density, safe UAM operations are possible if this new paradigm is developed for use in UAM air traffic management.

It is recommended that NASA and the commercial UAS developers consider simulation and further analytical development of the ABTM concept for use in potential UAM operations. This research could confirm or disprove its viability in this demanding trajectory management environment and possibly move the realization of the UAM potential many years sooner.

\section{References}

[1] I. M. Gregory, "Self-Aware Vehicles for Urban Air Mobility - Challenges and Opportunities," in AFOSR Dynamics and Control Program Annual Reviewq, Arlington, VA, 2017.

[2] I. M. Gregory, C. Leonard and S. J. Scotti, "Self-Aware Vehicles: Mission and Performance Adaptation to System Health," in 16th AIAA AVIATION Conference, Washington, DC, 2016.

[3] USADATA, "Dallas Fort Worth Arlington Metroplex," 2018. [Online]. Available: https://www.usadata.com/. [Accessed 26 April 2018].

[4] G. Norris, "Safety First," Aviation Week \& Space Technology, 29 January 2018.

[5] W. B. Cotton, R. Hilb, S. Koczo and D. J. Wing, "A Vision and Roadmap for Increasing User Autonomy in Flight Operations in the National Airspace," in 16th AIAA Aviation Technology, Integration, and Operations Conference, Washington, DC, 2016.

[6] W. B. Cotton, R. Hilb, S. Koczo and D. J. Wing, "Preliminary Assessment of Operational Hazards and Safety Requirements for Airborne Trajectory Management (ABTM) Roadmap Applications," NASA/TM-2016219176, Hampton, VA, 2016.

12

American Institute of Aeronautics and Astronautics 
[7] D. J. Wing and W. B. Cotton, "Autonomous Flight Rules: A Concept for Self-Separation in U.S. Domestic Airspace," NASA/TP-2011-217174, Hampton, VA, 2011.

[8] A. J. Hawkins, "EHang's Passenger-Carrying Drones Look Insanely Impressive in First Test Flights," The Verge, 5 February 2018.

[9] D. A. Karr, R. A. Vivona, D. A. Roscoe, S. M. DePascale and D. J. Wing, "Autonomous Operations Planner: A Flexible Platform for Research in Flight-Deck Support for Airborne Self-Separation," in 12th AIAA Aviation Technology, Integration, and Operations (ATIO) Conference, Indianapolis, AIAA-2012-5417.

[10] RTCA, Inc., "DO-365 - Minimum Operational Performance Standards (MOPS) for Detect and Avoid (DAA) Systems," RTCA, Washginton DC, 2017.

[11] C. Munoz and A. Narkawicz, "Formal Analysis of Extended Well-Clear Boundaries for Unmanned Aircraft," in 8th NASA Formal Methods Symposium, Washington, DC, 2016.

[12] D. Wartofsky, "Can Artificial Intelligence Replace Human Controllers?," Journal of Air Traffic Control, ATCA, vol. 59, no. 2, 2017. 